\title{
Vogelbestäubung bei südafrikanischen Salbei-Arten
}

\author{
Petra Wester
}

\begin{abstract}
Most bird-pollinated species of the large, otherwise mostly bee-pollinated genus Salvia are neotropical in distribution. Until recently, only one Old World species was known to be pollinated by birds, Salvia africana-lutea from South Africa. Based on their floral characters, two additional species, S. lanceolata and S. thermarum, were recently shown to be birdpollinated as well. For the first time, Sunbirds and White-eyes have been observed pollinating flowers of these species in the Kirstenbosch Botanical Garden in Cape Town.
\end{abstract}

\section{Zusammenfassung}

In der vorwiegend bienenbestäubten Gattung Salbei (Salvia) kommen fast alle vogelbestäubten Arten in der Neuen Welt vor. Bis vor kurzem war nur eine einzige altweltliche Art als vogelblütig bekannt, nämlich Salvia africana-lutea aus Südafrika. Kürzlich wurden zwei weitere Arten, S. lanceolata und S. thermarum, aufgrund ihrer Blütenmerkmale als vogelblütig identifiziert. Im Botanischen Garten Kirstenbosch (Kapstadt) konnten Nektar- und Brillenvögel erstmalig beim Bestäubungsvorgang beschrieben und illustriert werden.

\section{Einführung}

Die weltweit verbreitete, vor allem von Bienen bestäubte Gattung Salvia (Salbei, Lamiaceae) umfasst annähernd 1000 Arten, von denen ungefähr 350 in der Alten Welt vorkommen. Während es in der Neuen Welt etwa 180 von Vögeln bestäubte Arten gibt, die überwiegend von Kolibris aufgesucht werden, ist in der Alten Welt nur eine einzige vogelblütige Art bekannt, nämlich die südafrikanische Salvia africanalutea.

\section{Salvia africana-lutea: über 100 Jahre ein- ziger bekannter vogelbluitiger Salbei der Alten Welt}

Salvia africana-lutea $(=S$. aurea) ist auch unter den Namen Strand-Salbei oder Gold-Salvia bekannt. Die Art kommt vom Namaqualand bis zur Kap-Halbinsel und weiter östlich bis Port Alfred vor. Dieser Salbei wächst auf Küstendünen und bis $800 \mathrm{~m}$ Höhe auf steinigen Hängen im trockenen Fynbos, einer sehr artenreichen Vegetationseinheit im Kapland (Hedge 1985). Strand-Salbei ist ein reich verzweigter Strauch,

Abb. 1 (oben): Blüte von Salvia africana-lutea mit zurückgebogener Unterlippe

Abb. 2 (unten): Pollentransfer in der Gattung Salvia mit Hilfe des Staubblatthebels. Der Blütenbesucher drückt den unteren Hebelast nach hinten und löst dabei den Hebelmechanismus aus, so dass der Pollen auf seinem Kopf abgelegt wird.
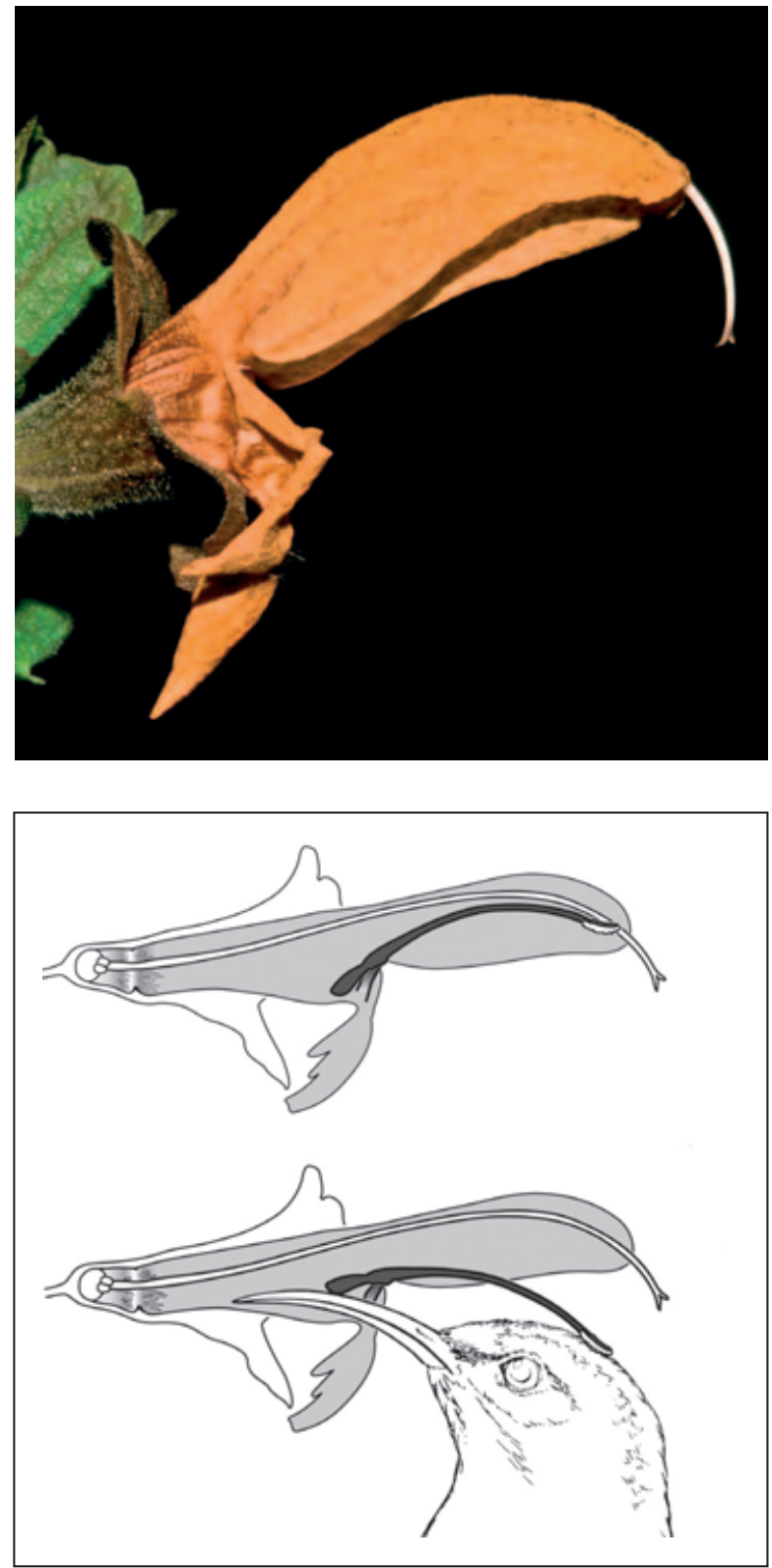


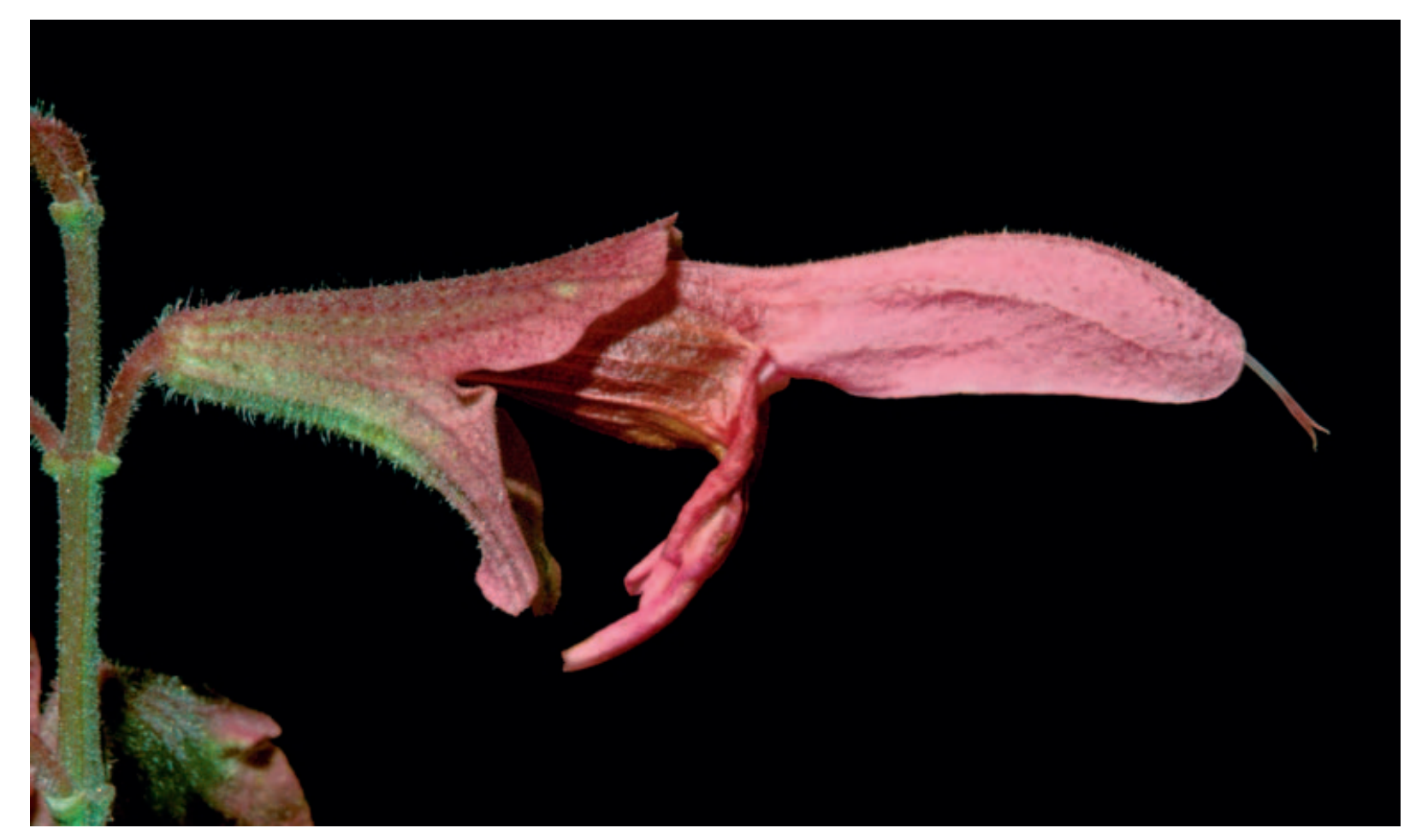

der bis zwei Meter hoch wird. Seine Blüten deuten auf Vogelbestäubung hin (Vogel 1954, Wester \& Classen-Bockhoff 2006). Sie sind mit einer Länge von ca. $5 \mathrm{~cm}$ relativ groß und auffällig gelblich-braun gefärbt (Abb. 1). Ihre zurückgebogene Unterlippe bietet Insekten keinen Landeplatz, erleichtert Vögeln aber das Nektartrinken. Die Blüten enthalten reichlich schwach konzentrierten Nektar, der manchmal die gesamte Blütenröhre füllt und aus dem Blüteneingang hinaustropft.

Vor über 100 Jahren wurden erstmals blütenbiologische Beobachtungen an dieser Art dokumentiert. Im Botanischen Garten von Kapstadt (heute Botanischer Garten Kirstenbosch) beobachtete der britische Soldat und Botaniker George Francis Scott-Elliot (1890) den Kap-Brillenvogel (Zosterops pallidus) beim Besuch der Blüten von Salvia africana-lutea. Insekten sah er an den Blüten nie. Sсотт-ElLiot beschrieb auch den spektakulären Mechanismus der Pollenübertragung, wie er für die Gattung Salbei (Classen-Bockhoff et al. 2003) typisch ist. Spätere Beobachtungen ergaben, dass S. africanalutea auch vom Großen und Kleinen HalsbandNektarvogel (Nectarinia afra und N. chalybea) bestäubt wird (SCHMIDT 1964, BRIESCHKE 1991).
Die beiden Staubblätter in der Salbei-Blüte sind zu Hebeln umgebildet (Abb. 2). Vögel oder Insekten, die nach Nektar suchen, müssen die beiden unteren Hebelarme, die den Blüteneingang und somit den Zugang zum Nektar blockieren, wegdrücken. Dabei gleiten die Pollensäcke am Ende der oberen Hebelarme aus der Oberlippe hervor und werden auf den Körper des Bestäubers gedrückt. Besucht letzterer anschließend eine weitere Blüte der gleichen Art, wird die Übertragung des Pollens auf die Narbe des herausragenden Griffels ermöglicht.

\section{Salvia lanceolata und S. thermarum - erst kürzlich als vogelblütig erkannt}

Von den 23 südafrikanischen Salbei-Arten (Hedge 1985, VAN JaARSVELd 2002b) konnten zwei weitere Arten (S. lanceolata und S. thermarum) als vogelblütig identifiziert werden (WESTER \& Classen-Bockhoff 2006). Beide Arten haben große, ungefähr $4 \mathrm{~cm}$ ( $S$. lanceolata) bzw. fast $6 \mathrm{~cm}$ (S. thermarum) lange und auffällig gefärbte Blüten. Bei Salvia lanceolata sind sie pink-violett-purpurn gefärbt (Abb. 3), während diejenigen von $S$. thermarum rot sind (Abb. 4,

Abb. 3: Salvia lanceolata - auffällig rosa gefärbte Blüte. 


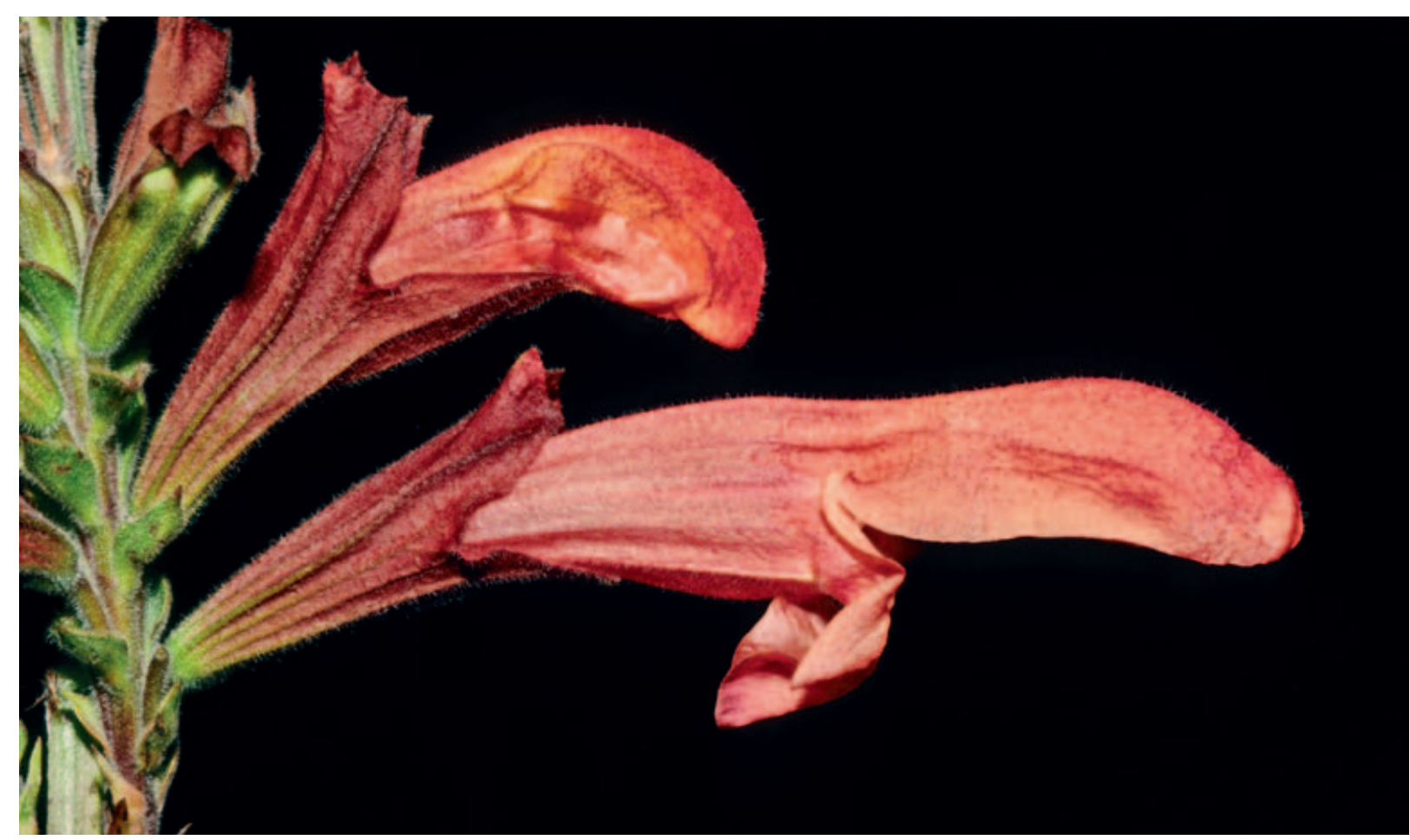

Wester \& Classen-Bockhoff 2006). In Kultur (im Botanischen Garten Kirstenbosch oder im Botanischen Garten der Universität Mainz) können sie auch blassrot bis pink oder lachsfarben gefärbt sein. Im Gewächshaus kultivierte Individuen entwickeln mitunter gelbliche Blüten. Die Unterlippen der Blüten beider Arten sind im Vergleich zu bienenblütigen Arten reduziert. Sie sind schmal bei $S$. lanceolata und kurz bei $S$. thermarum. Bei beiden Arten sind sie nach hinten umgebogen, so dass Bienen nicht oder nur schlecht auf der Unterlippe landen können. Die engen Blütenröhren sind lang (besonders bei $S$. thermarum), so dass die Mundwerkzeuge der Bienen im Gegensatz zu den langen Schnäbeln der Vögel zu kurz sind, um an den Nektar zu gelangen.

Salvia lanceolata (Lanzett-Salbei) ist ein hoher verzweigter Strauch, der vom Namaqualand bis zur Kap-Halbinsel und ostwärts bis Montagu Fynbos-Standorte bis in $300 \mathrm{~m}$ Höhe besiedelt (Hedge 1985). Salvia thermarum (GoudiniSalbei) wurde erst kürzlich in Goudini Spa nahe Worcester (West-Kap) entdeckt (vAN JAARSVELD 1999, 2002a, 2003). Es handelt sich um eine

Abb. 4: Salvia thermarum - große rote Blüte mit langer Kronröhre. mehrjährige Staude, die bis ungefähr einen Meter hoch wird und im Proteaceen-Fynbos wächst. Der Artname thermarum deutet auf die heißen Goudini-Quellen.

Im Januar 2004 und Oktober 2005 wurden beide Geschlechter des Kleinen Halsband-Nektarvogels und des Kap-Brillenvogels an Blüten von S. lanceolata im Botanischen Garten Kirstenbosch in Kapstadt beobachtet (Wester \& Classen-Bockhoff 2006). Der Kleine Halsband-Nektarvogel besuchte auch die Blüten von S. thermarum. Die Vögel saßen an den Ästen des Strauches und führten ihre Schnäbel in die Blüten ein und zogen die Blüten zu sich heran. Dieses Verhalten wird durch die langen und flexiblen Blütenstiele ermöglicht. Während die Vögel ihre Schnäbel in die Blüten einführten, drückten sie den unteren Hebelast nach hinten und wurden auf dem Kopf mit Pollen eingestäubt.

\section{Mögliche Hybridisierung}

Da die drei Arten zumindest im Kleinen Halsband-Nektarvogel einen gleichen Bestäuber haben und sich die Verbreitungsgebiete im West-Kap großflächig überlappen, sind natürliche Hybriden zu erwarten. Es wurden Individuen mit intermediären Merkmalen gefunden. 

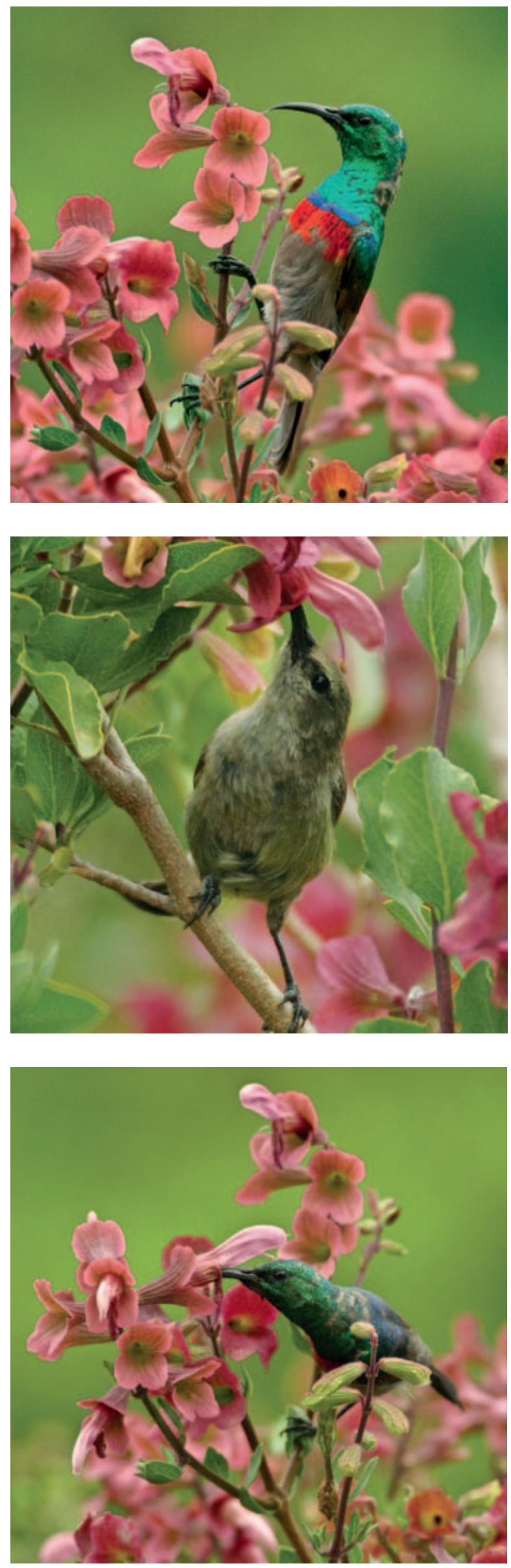

Dies äußerte sich z. B. in der Farbe und Größe der Blüten von Hybriden zwischen S. africanalutea und S. lanceolata (Wester \& ClassenBocKHOFF 2006). Man vermutet auch Hybriden zwischen der südafrikanischen $S$. albicaulis und S. granitica sowie zwischen $S$. lanceolata und $S$. africana-caerulea (VAN JAARSVELD 2002b).

\section{Dank}

Mein Dank gilt Rafael Groneberg (Mainz) für die Abbildungen.

\section{Literatur}

BRIEschKe, H. 1991: Brutbiologische und nahrungsökologische Untersuchungen am Kleinen Halsbandnektarvogel (Panaeola chalybea) und am Großen Halsbandnektarvogel (Panaeola afra) im Freiland (Südafrika) und im Labor. - Dissertation, Universität Frankfurt. Classen-Bockhoff, R., Wester, P. \& Tweraser, E. 2003: The staminal lever mechanism in Salvia - a review. Plant Biology 5: 33-41.

Hedge, I. C. 1985: Salvia. - Flora of Southern Africa 28: 79-101.

Schmidt, R. K. 1964: The Lesser Double-collared Sunbird, Cinnyris chalybeus (Linnaeus) in the southwestern Cape. - The Ostrich 35: 86-94.

Scott-Elliot, G. F. 1890: Ornithophilous plants in South Africa. - Ann. Bot. 4: 265-280.

VAn JaARsveld, E. J. 1999: Salvia thermara, a new species from the Western Cape, South Africa. - Bothalia 29: 100-102.

VAN JAARSVELD, E. J. 2002a: The Goudini sage. Veld \& Flora 88: 66-67.

VAn JAARsveld, E. J. 2002b: South African sages. Veld \& Flora 88: 102-104.

Van JaArsveld, E. J. 2003: The correct name for Salvia thermara. - Bothalia 33: 112.

Vogel, S. 1954: Blütenbiologische Typen als Elemente der Sippengliederung dargestellt anhand der Flora Südafrikas. - Bot. Stud. 1: 1-338.

Abb. 5 (oben): Ein Männchen des Kleinen Halsband-Nektarvogels im Brutkleid im Botanischen Garten Kirstenbosch, sitzend auf einem Ast von Salvia lanceolata.

Abb. 6 (Mitte): Salvia lanceolata wird von einem Weibchen des Kleinen Halsband-Nektarvogels besucht. Nachdem der Schnabel in die Blüte eingeführt wird, senkt sich der vordere Staubblatt-Hebelast.

Abb. 7 (unten): Ein Männchen des Kleinen HalsbandNektarvogels an einer Blüte von Salvia lanceolata. Die Pollensäcke berühren den Kopf des Vogels. 